\title{
Aplicação do método de inventário geobotânico para análise de formações vegetais fanerófitas e caméfitas na Serra de Caturité, semiárido da Paraíba - Nordeste do Brasil
}

Inventory method of application geobotanic for formations analysis of phanerophytes and caméfitas vegetables in Serra de Caturité, semiarid of Paraíba - northeastern Brazil

\author{
LIMA $^{1}$, V. R. P.; ARTIGAS ${ }^{2}$, R. C.
}

vrportol@uepb.edu.br

\begin{abstract}
Resumo
$\mathrm{O}$ artigo possui o objetivo de apresentar uma proposta metodológica para realizar inventários geobotânicas, desenvolvido com a finalidade de suprir algumas lacunas observadas em propostas metodológicas de inventários fitossociológicos. O Método de Inventários para Espécies Fanerófitas e Caméfitas (MIFC) é uma modificação da metodologia de Gentry (1982) que, associada a aspectos da ecodinâmica, possibilita o desenvolvimento de uma análise mais dinâmica e menos descritiva das formações vegetais. Nesse sentido, é possível analisar a biodiversidade local e caracterizar unidades fisionômicoestruturais-fenológicas, considerando fatores mesológicos das formações superficiais e condições bioclimáticas. A pesquisa foi realizada no inselberg Serra de Caturité localizado na Microrregião do Cariri Oriental no semiárido Paraibano. Os resultados aqui apresentados possibilitou classificar três biótipos de vegetação de caatinga com condição bioclimática tropófila semiárida, quatro meses de paralisação vegetativa, onze meses de déficit hídrico e apenas um mês de recarga de água no solo, variando em cada biótipo os aspectos relacionados à estrutura, altura, densidade, cobertura e diversidade de espécies nas vertentes baixa, media, e média-alta do inselberg.
\end{abstract}

Palavras-chave: Caatinga, Biogeografia, Metodologi.

\begin{abstract}
This article has the objective of presenting a proposal for methodological application to carry out geobotânicas inventories, developed in order to fill some gaps observed in methodological proposals phytosociological inventories. The Inventory Method for phanerophytes and Caméfitas Species (IMPC) is a modification of the Gentry methodology (1982) that associated with aspects of ecodynamics, allows the development of a more dynamic analysis and less descriptive of vegetation formations. Therefore, it is possible to analyze the local biodiversity and characterize physiognomic-structural-phenological units, mesológicos considering factors of surface formations and bioclimatic conditions. The survey was conducted in inselberg Serra de Caturité Microregion located in the Eastern of Cariri in the semiarid region of Paraíba. The results presented below make it possible classify three biotypes caatinga vegetation with bioclimatic condition tropófila semiarid four months of downtime vegetative, eleven months of drought and only a month to recharge the ground ranging in each biotype aspects related to the structure, height, density, coverage and diversity of species in the lower slopes, medium and medium-high of inselberg.
\end{abstract}

Keywords: Caatinga , Biogeography, methodology

\section{INTRODUÇÃO}

A Biogeografia enquanto disciplina da Geografia Física, foi introduzida nos meios acadêmicos no fim do século XIX. Seus ensinamentos estiveram vinculados às teorias das ciências naturais da fauna - zoogeografia e da flora terrestre - fitogeografia. Durante anos, as aplicações metodológicas para estudos em fitogeografia estiveram vinculadas aos levantamentos fisionômicos e fitossociologicos. Tais aplicações resultaram em trabalhos de descrições da vegetação, ou ainda, apenas do conhecimento empírico da flora (Giehl, Budke, 2011).

A fitossociologia contou com grande contribuição do método de Braun-Blanquet (1950), que orienta até a atualidade diversas disciplinas que realizam estudos estruturais e florísticos. A 
fitossociologia difundida por Braun-Blanquet foi desenvolvida para ser aplicada em países de clima temperado, por isso, sua aplicação em meios tropicais enfrenta algumas limitações. Frente a essas limitações, surgiram outras propostas metodológicas para estudos em bosques tropicais, como exemplo, o método de Gentry (1982).

A proposta metodológica de Gentry para estudos fitogeograficos considera todos os indivíduos de espécies fanerófitas cujo diâmetro na altura do peito (DAP) seja superior a 2,5 cm, localizado dentro de um perímetro definido de $1 \mathrm{~m}$ a cada lado de uma linha de $50 \mathrm{~m}$. A análise dos dados permite estudar a diversidade de uma formação vegetal tomando como referência as espécies de tipo arbóreo em sua estrutura vertical, porém não considera a estrutura horizontal, e as espécies fanerófitas que possuem DAP inferior a $2,5 \mathrm{~cm}$. Neste sentido, sua aplicação direta para os bosques secos tropicais e para as formações arbustivas não é eficaz.

Com o objetivo de suprir as carências encontradas nas propostas metodológicas citadas, o Grupo de Estudo Tropicales y Biogeografia da Universidade de Sevilla - ES adaptou a metodologia desenvolvida por Gentry para desenvolver inventários de formações vegetais fanerófitas e caméfitos em bosques secos tropicais e mediterrâneos. A aplicação da metodologia MIFC vem sendo sistemática realizada em pesquisas desenvolvidas no Parque Nacional Doñana - ES, (Bejarano et. al., 2010) e foi aplicada pela primeira vez no Brasil em áreas de Caatinga no Estado da Paraíba na pesquisa desenvolvida por Lima, 2012.

Portanto, esse artigo tem por objetivo apresentar aplicação da metodologia e seus resultados alcançados no inselberg Serra de Caturité, localizado no município de Caturité, Microrregião do Cariri Paraibano.

\section{METODOLOGIA}

A metodologia de Inventario de Fanerófitos e Caméfitos (MIFC) consiste na aplicação de um conjunto de técnicas de campo para coleta de dados geobotânicas e no manuseio dos dados em planilhas automatizadas denominada Técnicas de Amostragem de Fanerófitos e Caméfitos em Transectos Lineares (TEFA), cujo acesso as planilhas podem ser encontradas na página: http://personales.us.es//rcamara/index archivos/mifc.htm

$\mathrm{Na}$ aplicação do MICF são realizados transectos lineares medindo 50X2m definidos por uma fita métrica. No transecto são identificadas todas as espécies e sua posição na distância longitudinal, como também a $1 \mathrm{~m}$ à direita e a esquerda de cada eixo da fita. Ainda são coletados dados da estrutura vertical, horizontal e altura de todos os indivíduos lenhosas arbustivas e subarbustivas, 
diâmetro maior e menor, caso a espécie não possua um DAP igual ou superior a $2 \mathrm{~cm}$; e para espécie que possuem um DAP igual ou superior a $2 \mathrm{~cm}$, são registrados o diâmetro de cada caule, o raio maior e menor da copa de cada individuo.

As parcelas podem ser realizadas paralela uma das outras ou em transectos contínuos que defina uma unidade homogênea de formação vegetal e formação geomorfológica. Com relação à quantidade de parcelas realizadas, seguimos a proposta de Gentry e postulada por Preston (curva área-espécies), que são dez parcelas para obter uma mostra de 0,1 hectares, para serem comparáveis independentes de suas características.

O levantamento também pode ser realizado de forma que marque uma subunidade geobotânica. Dessa forma, as parcelas são realizadas de forma linear, cobrindo as mudanças de tipos de solos, as formações superficiais ou de posição geomorfológica, sendo o número de parcelas realizado em função das mudanças que se deseja representar.

Os dados obtidos com a tabulação no TEFA podem ser analisados em quatro tipos de resultados:

$\checkmark$ Características dos atributos das unidades amostradas e seus elementos;

$\checkmark$ Características estruturais das formações vegetais;

$\checkmark$ Índices de diversidade $\alpha$ e $\beta$,

$\checkmark$ Tratamento estatístico multivariante das parcelas amostradas, segundo uma analise cluster, realizado no programa estatístico Past;

Esses resultados podem capturar cinco tabulações de dados agrupados em seções de $5 \mathrm{~m}$ dentro de cada transecto linear:

$\checkmark$ Tabulação 1: numero de indivíduos por espécie a cada $5 \mathrm{~m}$ e do total do transecto;

$\checkmark$ Tabulação 2: cobertura de espécies a cada $5 \mathrm{~m}$ e do total do transecto;

$\checkmark$ Tabulação 3: número de indivíduos por espécies e por estrado vertical a cada $5 \mathrm{~m}$ e do total do transecto;

$\checkmark$ Tabulação 4: cobertura de espécie por estrato vertical a cada $5 \mathrm{~m}$ e do total do transecto;

Tabulação 5: cobertura do DAP por espécie a cada $5 \mathrm{~m}$ e no total do transecto.

Os resultados relacionados à riqueza de espécies dos indivíduos são analisados através dos atributos: altura, cobertura, biótipo e DAP e sua caracterização a partir do Índice de Valor de Importância (IVI).

A estrutura horizontal (cobertura) é demonstrada através do Diagrama de Bolhas. A representação gráfica da cobertura das espécies identificadas é calculando através da superfície coberta segundo a área da elipse com o produto dos semieixos pelo numero de $\pi\left(a^{*} b^{*} \pi\right)$, ou a área da copa representando os arbustos e arvores (DAP) a partir do raio médio. 
Este tratamento é representado por três colunas para todas as espécies:

$\checkmark-\mathrm{X}=\mathrm{D}$ (distância em um transecto linear);

$\checkmark$ - Y=d (soma de colunas dd-di, distância a direita ou a esquerda do ponto D do transecto linear);

$\checkmark$ - R, se calcula do raio da bolha a partir da cobertura $R=(\text { cobertura } / / \pi)^{\wedge}(1 / 2)$.

Para o inselberg Serra de Caturité, foram realizados treze transectos com a finalidade de analisar subunidades geobotânicas, observando as mudanças de tipos e uso do solo e posição geomorfológica. Foram realizados três transectos na base do inselberg, oito na vertente média e dois na vertente alta.

Para melhor compreender a dinâmica da vegetação é preciso analisar alguns aspectos, entre eles balaços hídricos $(\mathrm{BH})$ e bioclimáticos (BB). Para o desenvolvimento desta etapa foi adotado o método de Regimes Ecodinamicos (MRE) desenvolvido por Cámara (1997), que busca associar as formações vegetais ao piso bioclimatico correspondente. O MRE, parte da base metodológica de Thornthwaite-Matter (López Cadenas, 1986) para o balanço hídrico e para o balanço bioclimático de Montero de Burgos y González de Rebollar (Montero de Burgos, 1974). Para elaboração dos gráficos de BH e BB acesse: http://personales.us.es//rcamara/index archivos/merec.htm

A análise dos dados paramétricos representados por gráficos de balanço hídrico $(\mathrm{BH})$ possuem três variáveis: pluviometria, evapotranspiração real e potencial, enquanto o balanço bioclimático (BB) apresenta duas variáveis que são a intensidade bioclimática potencial (IBP) e intensidade biclimática real (IBR). Os dados usados para gerar os $\mathrm{BH}$ e BB, do Município de Caturité, foram coletados do Banco de Dados Climáticos do Brasil da EMBRAPA - Monitoramento por Satélite.

\section{RESULTADOS E DISCUSSÃO}

A riqueza especifica encontrada na Serra de Caturité foi de 579 indivíduos, pertencentes a 18 famílias, 37 gêneros e 46 espécies. As espécies com maior índice de valor de importância (IVI) foram: Mimosa ophthalmocentra Mart. ex Bent, Poincianella pyramidalis (Tull) L.P. Queiroz. (Fabaceae) e Capparis flexuosa (L.) L. (Capparaceae). As famílias Euphorbiaecae e Fabaceae foram as mais representativas, com 10 e 9 espécies identificadas respectivamente.

A espécie Mimosa ophthalmocentra Mart. ex Benth. nome popular jurema-de-imbira, é secundária, estabelecendo-se em áreas antropizadas, apresenta uma grande amplitude de tolerância aos diferentes parâmetros físico-químicos do solo (Camargo-Ricalde, 2000), além 
desse fato, essa espécie possui um forte potencial madeireiro para o nordeste (Figueirôa et al. 2005).

A sistematização para análise dos dados foi realizada com agrupamento das parcelas em um Clusters, com o objetivo de identificar as espécies similares com o coeficiente de dados quantitativos de Morisita. Os resultados podem ser observados na figura 1.

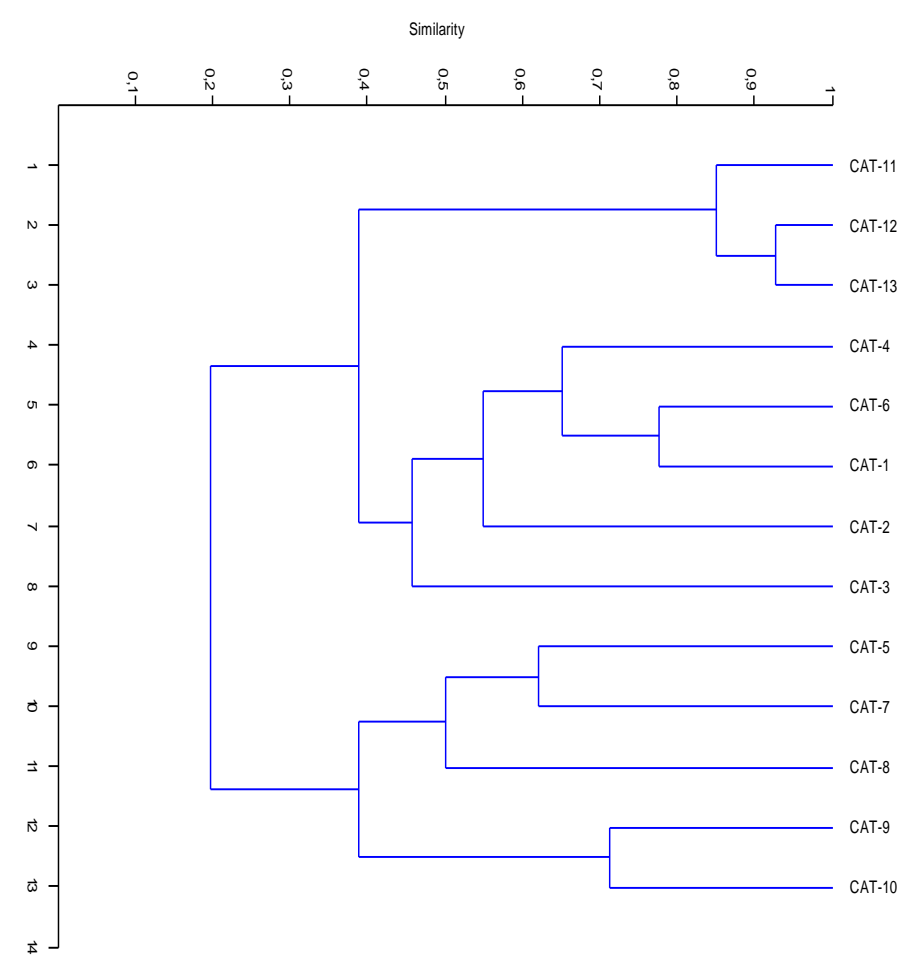

Figura 01. dendograma de classificação por analise de agrupamento das 13 parcelas de Caatinga na Serra de Caturité produzido a partir dos índices de Morisita (quantitativo). Fonte: Valéria Raquel Porto de Lima

A análise Clusters agrupou as parcelas por semelhança florística em três grupos, CAT 11, 12,13, referentes à vertente baixa, CAT 4, 6, 1, 2,3 referentes à vertente média e CAT 5, 7, 8, 9, 10 referentes à transição entre vertente media/alta. O agrupamento das espécies por similaridade florista obedece ao gradiente da posição geomorfológica da serra de Caturité, portanto demonstra uma influência direta da altitude na presença das espécies identificadas.

A distribuição e frequência do diâmetro na altura do peito (DAP) de todas as espécies mostra uma população dominante (moda) (gráfico 1), com maior concentração de espécies com DAP em torno dos 10cm, seguidos dos exemplares com 5 e $15 \mathrm{~cm}$. Existe uma diferença significativa entre os elementos de $5,10,15 \mathrm{~cm}$ para os demais, o que pode estar relacionado com o manejo recente do boques, por isso, o mesmo encontra-se em um estádio de sucessão secundária, com um número não muito elevado de espécies arbóreas altas. 
A estrutura vertical, representada nos gráficos de bolhas, mostra a distribuição e cobertura das espécies ao longo da transecto. Na vertente baixa, no transecto CAT-13, a espécie com maior abundância é a Poincianella pyramidalis (Tull) L.P. Queiroz com 23 indivíduos. A área basal total do transecto é $0,34 \mathrm{~m}^{2}$, a dominância relativa da Mimosa ophthalmocentra Mart. ex Bent, é de 59,91\% e área basal de 0,20m² (gráfico 1).

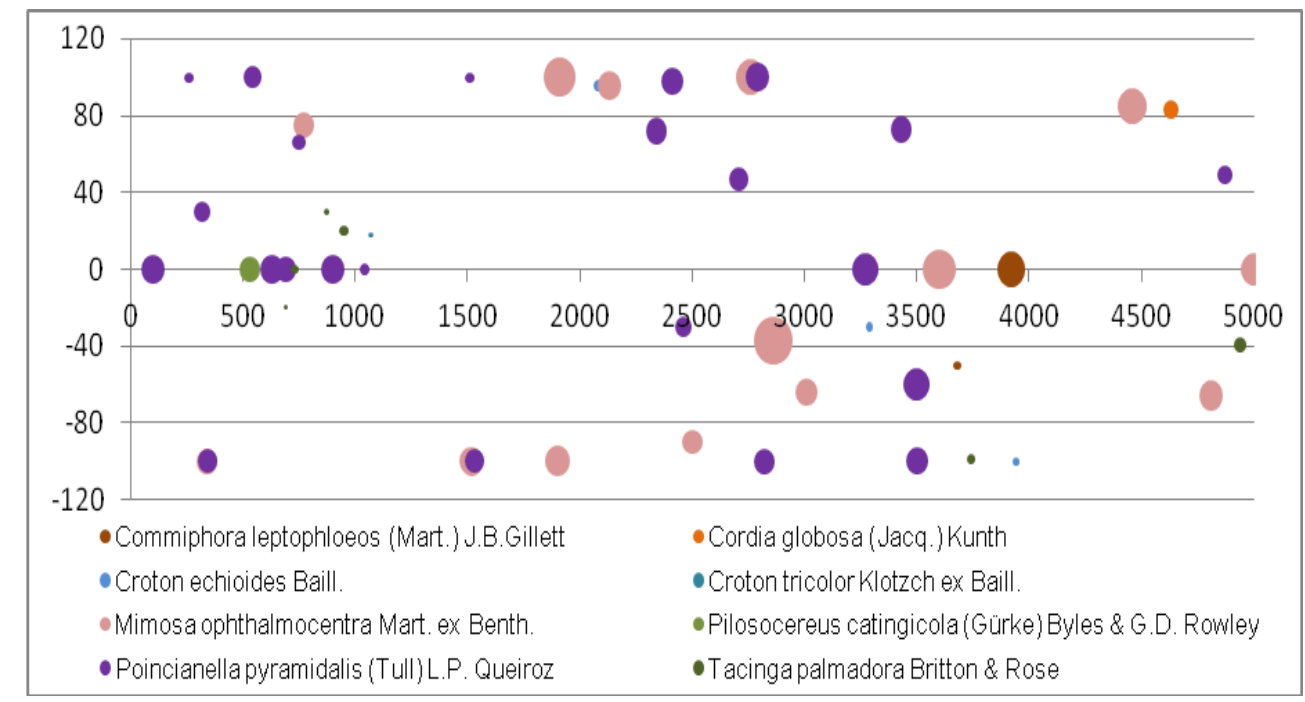

Figura 01. Gráfico representando a distribuição das espécies no transecto CAT 13 e sua área basal. Fonte: Valéria Raquel Porto de Lima (2012.)

Na verte media, transecto CAT - 4, a espécie com maior abundância é a Mimosa ophthalmocentra Mart. ex Bent, com dominância relativa de $24,70 \%$ e área basal de $0.13 \mathrm{~m}^{2}$ (gráfico 2).

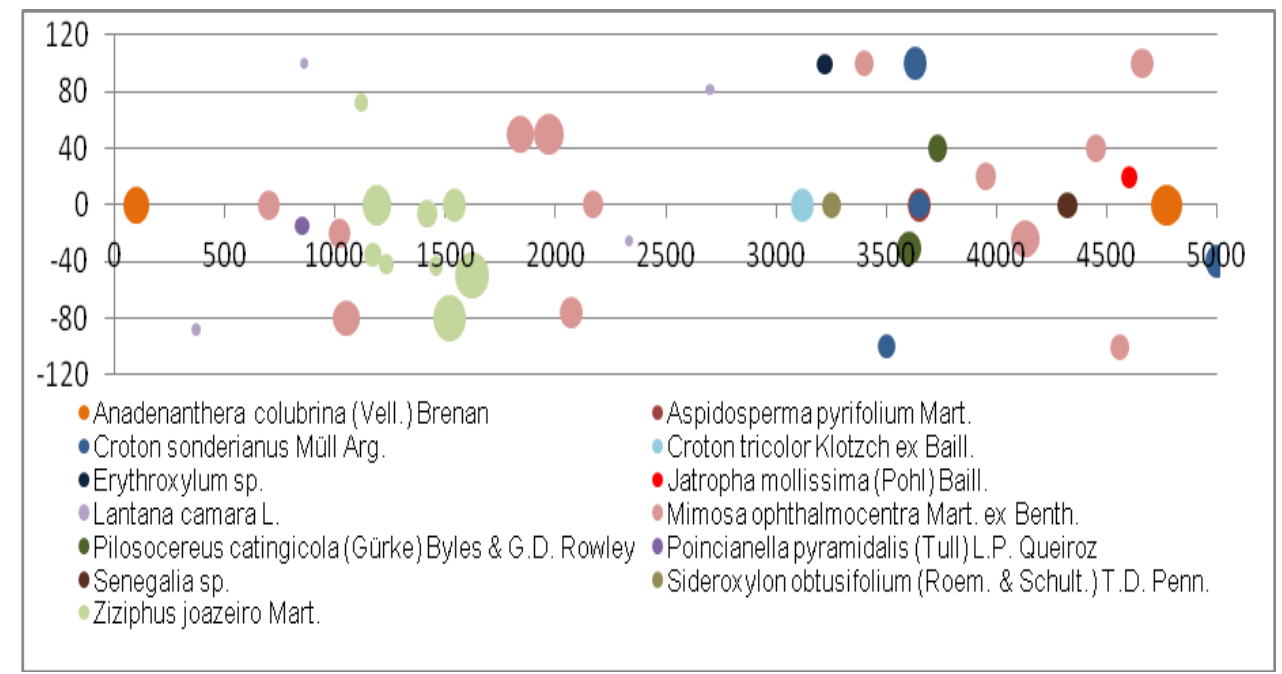

Figura 02. Gráfico representando a distribuição das espécies no transecto CAT 4 e sua área basal. Fonte: Valéria Raquel Porto de Lima (2012.) 
$\mathrm{Na}$ vertente alta, representada na parcela CAT - 7, existe uma maior abundancia da Capparis flexuosa (L.) L. As espécies Ceiba glaziovii (Kuntze) K. Schum e Syagrus oleracea (Mart.) Becc. foram identificadas apenas na vertente alta do inselberg. A área basal total do transecto é de 5,96 $\mathrm{m}^{2}$, desse total Pseudobombax marginatum (A. St.-Hil., Juss \& Cambess) A. Robyns, tem $5,60 \mathrm{~m}^{2}$ de área basal (gráfico 3).

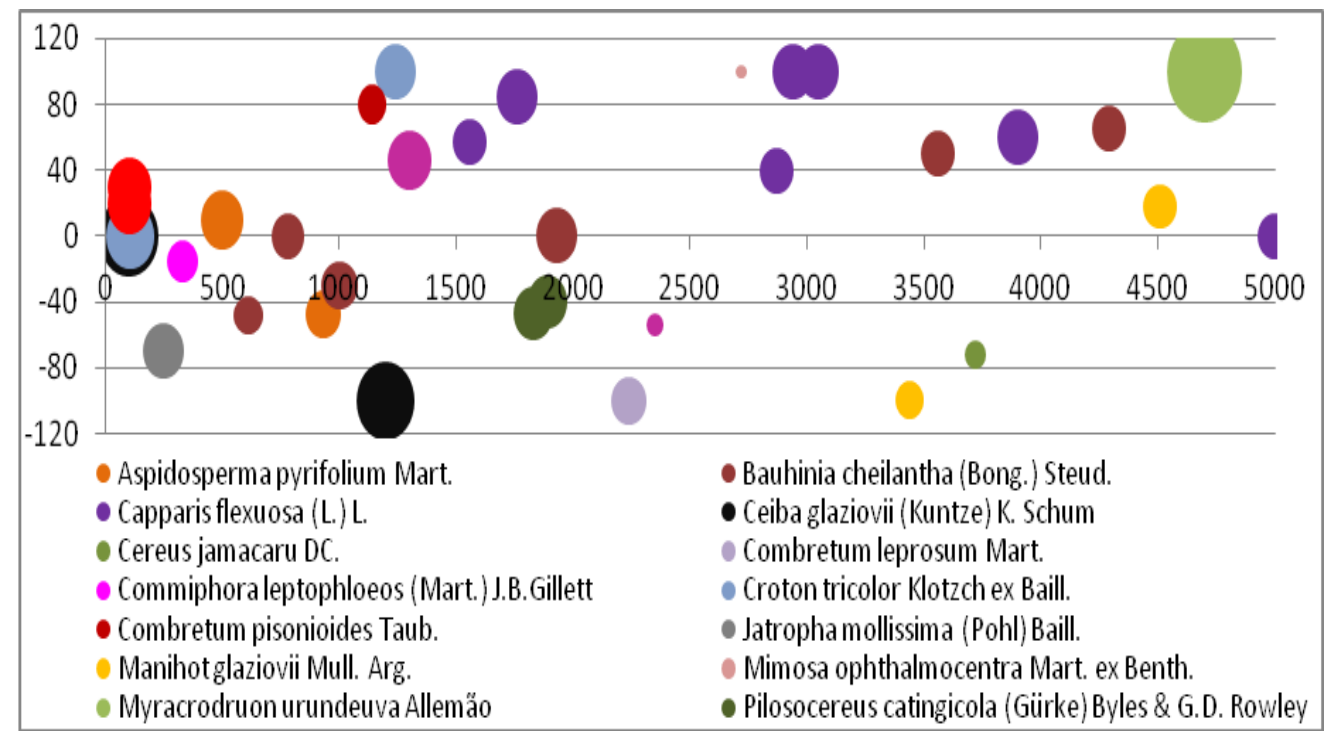

Figura 03. Gráfico representando a distribuição das espécies no transecto CAT 7 e sua área basal. Fonte: Valéria Raquel Porto de Lima (2012.)

Do conjunto de bosques tropicais e subtropicais do mundo, os bosques secos estacionais representam um total de 42\%. Estes bosques, segundo Pennington et al. (2000), se distribuem nas áreas da América Central e do Caribe, costa caribenha da Colômbia, costa peruana equatoriana, vales andinos equatorianos, peruanos e bolivianos, a oeste do México, em núcleos localizados no Panamá (arco seco) e nas Caatingas do Nordeste do Brasil. Em geral os bosques secos tropicais estacionais, apresentam a metade ou um terço do total de espécies lenhosas e possuem menor diversidade se comparados com os bosques úmidos tropicais, porém, em algumas situações podem apresentar índices de diversidade similares.

Durante anos acreditou-se que a Caatinga por apresentar uma fisionomia, em grande parte de sua área, de porte arbustivo e aspectos xerófilos, possuía baixa diversidade de fauna e flora. Entretanto, os estudos sobre a biodiversidade desse bioma realizados por Leal, (2005) apresentaram resultados contrários.

Confirmando as análises dos autores anteriormente citados, os dados da tabela 1, indicam que no fragmento da vegetal de caatinga no inselberg Serra de Caturité, o índice de ShannonWiener (H') é de 3,1 uma diversidade alta de espécies.

\begin{tabular}{l|l}
\hline Índices & Dados \\
\hline Shannon_H & $\mathbf{3 , 1 2 5}$ \\
\hline Equitability_J & $\mathbf{0 , 8 2 5 8}$ \\
\hline
\end{tabular}


Tabela 01. Dados de diversidade $\alpha$ para área de estuado.

Fonte: Valéria Raquel Porto de Lima (2012)

Pesquisas desenvolvidas por Alcoforado-Filho, et al. (2003) e Rodal, et al. (1998), encontraram valores de diversidade de 2,73 e 3,09 nats indivíduo-1 em áreas de caatinga com as mesmas características da área estudada.

As características da flora encontrada no local também estão relacionadas aos aspectos hídricos e bioclimáticos. A figura 4 indica a existência de um bosque tropófilo semiárido, com quatro meses de paralisação vegetativa, onze meses de déficit hídrico e apenas um mês de recarga de água no solo.

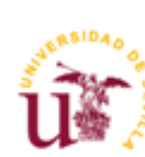

Diagrama de Balance Hidrico de Thornthwaite y Matter
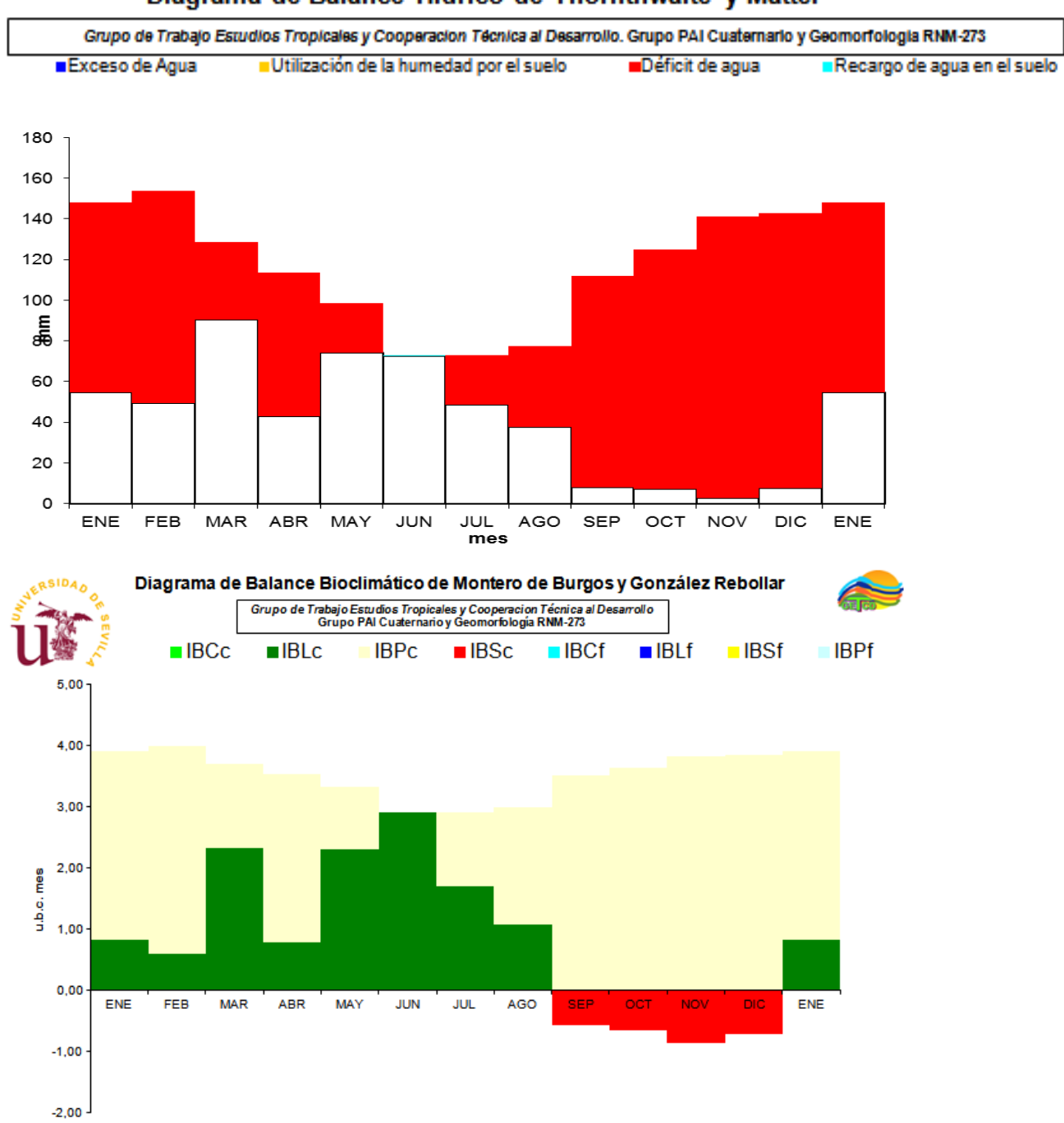

mes

Figura 04. Gráficos de balaço hídrico e bioclimático parao município de Caturité - PB.

Fonte: Valéria Raquel Porto de Lima (2012.) 
Dessa maneira, associando fatores hídricos, bioclimaticos e posição geomorfológica, as características estruturais, fisionômicas e de diversidade no local estão representadas em três biótipos, a saber: vertente baixa - boque tropófilo semiárido com pote arbustiva, alta densidade de cobertura. Vertente média - boque tropófilo semiárido de porte arbóreo, com alta densidade de cobertura a partir do extrato arbustivo alto com 1,5m a 3,5m; Vertente média/alta - boque tropófilo semiárido com porte arbóreo, alta densidade e diversidade de espécies. Certamente este biótipo encontra-se mais preservada devido a uma maior dificuldade de acesso a exploração da madeira nos partes mais elevadas do inselberg.

Em decorrência dos diversos tipos de manejo local ao longo dos anos, como o desmatamento para o plantio do algodão, atividade econômica dominante na região até as décadas de 1970 e 1980; o desmatamento para a venda da lenha para pólos gesseiros, cerâmicos e de padarias no estado da Paraíba e Pernambuco e o plantio da agricultura de subsistência a área apresenta uma vegetação de caatinga secundaria em processo de resiliência com aspectos visíveis de sucessão ecologia, sobretudo nas vertentes baixa e media.

\section{CONSIDERAÇÕES FINAIS}

A proposta metodológica possibilitou o preenchimento de algumas lacunas encontradas em metodologias de inventários fitosociológico, sobretudo pela possibilidade de inventariar espécies arbustivas como também as cactáceas e bromeliáceas representativas do bioma Caatinga. Os dados levantados permitiram o agrupamento de informações quantitativas que, trabalhados dentro de uma perspectiva sistêmica, e associados aos dados, biclimáticos e posição geomorfológica, possibilitaram uma análise qualitativa do comportamento estrutural e da biodiversidade da caatinga no semiárido no inselberg Serra de Caturité.

\section{REFERÊNCIAS}

ALCOFORADO-FILHO, F. G.; SAMPAIO, E. V. S. B; RODAL, M. J. N. Florística e Fitossociologia de um Remanescente de Vegetação Caducifólia Espinhosa Arbórea em Caruaru, $\begin{array}{llllll}\text { Pernambuco. Acta bot. } & \text { bras. }\end{array}$ http://www.scielo.br/pdf/abb/v17n2/a11v17n2.pdf Acceso en: 29/01/2012.

Bejarano, R. P., CÁMARA, R. A, BORJA C. B., DÍAZ, F. del Olmo, ReCiO. J. M. E. Caracterización de los Bosques de Sabina (Juniperus Turbinata Guss.) del Entorno de la Laguna de Charco del Toro (Parque Nacional Doñana, Huelva): Aplicación de una Nueva Metodología para el 
Estudio de la Vegetación. Biogeografía, una Ciencia para la Conservación del Medio. Compobell, S.L. Vol. 1. Pag. 25-34. Murcia. 2010.

BRAUN-BLANQUET, J. Sociología vegetal: estudios de las comunidades vegetales. Buenos Aires: Acme Agency, 1950. 444 p.

CAMARA, R. República Dominicana: dinámica del medio físico en la región Caribe (Geografía física, Sabanas y Litoral). Aportación al conocimiento de la tropicalidad insular. (Tese Doutorado). Programa de Doctorado en Geografía Física. Departamento de Geografía Física y Análisis Geográfico Regional. Universidad de Sevilla - Espanha. 1997.

CAMARGO-RICALDE, S.L. Descripción, distribución, anatomía, composición química y usos de Mimosa tenuifl ora (Fabaceae-Mimosoideae) em México. Revista de Biologia Tropical 48(4): 123. 2000.

FIGUEIRÔA, J.M.; PAREYN, F.G.C.; DRUMOND, M.; ARAÚJO, E.L. Madeireiras. Pp. 1011330. In: Sampaio, E.V.S.B.; Pareyn, F.G.C.; de Figueirôa J.M. \& Santos Jr. A.G. (Eds.) Espécies da flora nordestina de importância econômica potencial. Recife, Associação Plantas do Nordeste (APNE). 2005.

GENTRY, A.H. Diversity and floristic composition of Neotropical dry forests. In: BULLOCK, S.H., MOONEY. H.A. Seasonal dry tropical forests. Cambridge University Press, Londres. Ed. Medina. p. 146-190. 1995

GENTRY, A. H. Patterns of Neotropical plant species diversity. Evolutionary Biology 15:1-84. 1982.

GIEHL, E.L.H. \& BUDKE, J.C. Aplicação do método científico em estudos fitossociológicos no Brasil: em busca de um paradigma. Pp. 1-21. In: Felfi li, J.M.; Eisenlohr, P.V.; Melo, M.M.R.F. Andrade, L.A.; Meira Neto J.A.A. (Org.). Fitossociologia no Brasil: métodos e estudos de caso. UFV: Viçosa, Sociedade Botânica do Brasil. 2011.

LEAL, I. R; TABARELLI, M. SILVA, J. M. C.(org.), Ecologia e Conservação da Caatinga. Ed. Universidade Federal de Pernambuco. 20 Edição. 822pag. 2005.

LIMA, V. R. P. Caracterización Biogeográfica del Bioma Caatinga en el Sector Semiárido dela cuenca del río Paraíba: Propuesta de Ordenación y Gestión de un Medio Tropical Semiárido. Tese de Doutorado. Programa de Doctorado en Geografía Física. Departamento de Geografía Física y Análisis Geográfico Regional. Universidad de Sevilla - Espanha, 2012,

LÓPEZ CADENAS DE LLANO, F. y J.A. MINTEGUI AGUIRRE. Hidrología de Superficie. Tomo I. Ed. Fund.Conde Salazar. ETSI. Montes. Madrid. 1986.

MONTEIRO DE BURGOS, J.L. y REBOLLAR, J.L.G. Diagramas bioclimáticos. Ministerio de Agricultura, ICONA. Madrid. 1974.

PENNINGTON R.T., PRADO D.E., PENDRY. C.A. Neotropical Seasonally dry forests and quaternary vegetation changes. J. Biogeog. 27: 261-273. 2000. 
RODAL, M. J. N; ANDRADE, K. V. A; SALES, M. F; GOMES; A. P. S. Fitossociologia do Componente Lenhoso de um Refúgio Vegetacional no Município de Buíque, Pernambuco. Revista Brasileira Biologia. vol.58 no.3 São Carlos. ISSN 0034-7108. 1998.

\section{AGRADECIMENTOS}

Ao programa de pós graduação de Geografia da UFPB e a Agencia Española de Cooperación Internacional para el Desarrollo (AECID).

Recebido em: 14/08/2016

Aceito para publicação em: 01/10/2016 